
\title{
25 Research Soure \\ Continuing use of aspirin in total knee arthroplasty: safe or not?
}

\section{Zeng Li}

Guangdong Provincial People's Hospital, Guangdong Academy of Medical Sciences

\section{Shuai Xiang}

The Affiliated Hospital of Qingdao University

\section{Yan Du}

The Academy of Integrative Medicine of Fudan University

\section{Yanyan Bian}

Peking Union Medical College Hospital, Chinese Academy of Medical Science

\section{Bin Feng}

Peking Union Medical College Hospital, Chinese Academy of Medical Science

\section{Xisheng Weng ( $\nabla$ doctorwxs@163.com )}

Peking Union Medical College Hospital, Chinese Academy of Medical Science

\section{Research Article}

Keywords: Aspirin, total knee arthroplasty, blood loss, complication

Posted Date: May 6th, 2021

DOl: https://doi.org/10.21203/rs.3.rs-492900/v1

License: (c) (1) This work is licensed under a Creative Commons Attribution 4.0 International License. Read Full License 


\section{Abstract}

Background: There are a growing number of patients who take aspirin routinely before TKA to prevent vascular events. However, it may increase the risk of bleeding and wound complications. It remains controversial regarding the safety of continuing use of aspirin during the perioperative period of TKA.

Methods: The present study retrospectively selected TKA cases from the Joint Arthroplasty Database at the Peking Union Medical College Hospital from January 2014 to December 2019. The comparisons between the aspirin group and non-aspirin group on the aspects of bleeding parameters (blood loss, transfusion rate, drainage volume, hemoglobin and hematocrit change) and complications (cardiac events, venous thromboembolism events, cerebrovascular events and wound events) were performed.

Results: A total of 560 patients with unilateral TKA and 285 patients with bilateral TKA were extracted. Among those, 280 patients used aspirin preoperatively. No differences were found in demographic and surgical characteristics between the aspirin and non-aspirin group except for the proportion of coronary artery diseases $(p<0.05)$. For the outcomes, there were no significant higher blood loss in the surgery and transfusion rate during or after the surgery in the aspirin group, while the drainage in the aspirin group was higher than control group, especially for bilateral TKAs $(p=0.043)$. And the hemoglobin and hematocrit in POD5 in aspirin group were significant lower in both unilateral and bilateral TKAs. For complications, though there were lower vascular related complication rate in aspirin group after unilateral TKAs $(p=0.040)$, the wound event rate in aspirin group were higher than the control group $(p=0.049)$.

Conclusion: Continuing use of aspirin could prevent vascular related complications during the perioperative period of TKA. However, it might also increase the risk of bleeding and wound complications which we should pay attention to.

\section{Background}

Total knee arthroplasty (TKA) is considered as one of the most successful surgeries that can correct the deformity and restore the function of the knees [1]. With increasing life expectancy, a growing number of elderly patients who suffer from osteoarthritis and other joint diseases choose to undertake TKA to improve life quality [2-3]. However, many of them have various comorbidities which requires taking medications during the perioperative period [3].

Aspirin is a common anti-platelet agent which has been used to prevent vascular complications in patients with cerebrovascular diseases, coronary artery diseases, atrial fibrillation and peripheral vascular diseases by inhibiting thrombus formation [4-5]. It had been reported that about $30 \%$ adult population used aspirin for vascular events prevention in United States from 2012 to 2015 [5-6]. In our database, the prevalence of using aspirin when admitted to hospital was more than 15\% in all TKA patients from 2014 to 2019. It would be more in the osteoarthritis patients because of their older age [2]. Therefore, it is of great importance to manage the use of aspirin during the perioperative period of TKA. 
Continuing use of aspirin preoperatively could be considered as a two-edged sword. It was reported that aspirin may play an important role in decreasing the risk of cardiac events, stroke and other vascular diseases in the perioperative period [7-8]. However, it may also increase the blood loss perioperatively or influence the wound healing after surgery [8]. According to an evidence-based guideline of antithrombotic medications management, continuing aspirin in orthopedic procedure is likely to increase bleeding risk, which could also result in poor wound healing [8].

To the best of our knowledge, published studies regarding aspirin use in TKA are limited. Two previous studies tried to compare the blood loss and transfusion rate in TKA with aspirin or without aspirin $[9,10]$. However, neither of them included bilateral TKA patients nor compared complication outcomes. Meier et al compared the complication outcomes, but the sample size was too small (17 TKA patients with aspirin) [11]. The consistency of the groups was also difficult. The studies of Schwab et al and Chen et al failed to eliminate the significant differences on age or body mass index (BMI) between aspirin and nonaspirin group $[9,12]$. In the present study, we aimed to compare the blood loss, transfusion rate and complication rate of the unilateral and bilateral TKA between the aspirin group and non-aspirin group with more consistent criteria.

\section{Methods}

\section{Study design}

The present study retrospectively reviewed the TKA cases from the Joint Arthroplasty Database at the Peking Union Medical College Hospital from January 2014 to December 2019. This study was approved by the Research and Ethics Institutional Committee of Peking Union Medical College Hospital and performed in accordance with the relevant guidelines and regulations.

The inclusion criteria were: patients undertook primary unilateral TKA or bilateral TKA simultaneously without undergoing other surgeries in one admission; the main orthopedic diagnosis of the patients should be primary osteoarthritis; patients had comorbidity or history which indicated risk of vascular events according to the American College of Cardiology (ACC)/American Heart Association (AHA) guideline, such as diabetes, stroke, heart failure, valvular diseases, coronary artery diseases (CAD), and certain arrhythmias including ventricular arrhythmias, superventricular arrhythmias, high-grade atrioventricular block and atrial fibrillation [13]; tranexamic acid was administrated intravenously during surgery as routine and tourniquet and drainage were also used after surgery; all included cases were follow the standard rehabilitation schedule and thrombosis prevention program, including ankle flexion and extension, compression stockings and anticoagulation plan (low molecular heparin after surgery for 4 days and Rivaroxaban for 10 days, then continue to take aspirin if needed). We excluded patients with connective tissue diseases, cancers, blood diseases or using medications that may influence blood test results other than aspirin [14-15]. Cases were reviewed by two authors independently. The cases which had disagreement were screened by the third author to determine whether the cases were included or not. 
The included patients were categorized into aspirin group or non-aspirin group based on the use of aspirin preoperatively.

\section{Data Extraction}

The following patient information were extracted from medical records: demographic characteristics including age, sex, BMI; medical conditions including knee deformity, comorbidities, tobacco and alcohol use; surgical data including sides, anesthesia, implant type and tourniquet time. The primary outcome was bleeding risk, including blood loss and transfusion during the surgery, drainage, transfusion after the surgery, hemoglobin (HGB) and hematocrit (HCT) value before the surgery and at the first, second, third, fifth day after the surgery (POD 1, 2,3,5). The secondary outcomes were complications classified as cardiac events, venous thromboembolism events, cerebrovascular events and wound events. The followup was as least one year after surgery. All the data were extracted by two authors independently and supervised by a third author to guarantee the accuracy.

\section{Statistical analysis}

Continuous variables were presented as mean and standard deviation (SD). For normally distributed continuous variables, Student's unpaired t-test was used to compare the differences between two groups. For nonparametric variables, Mann-Whitney U-test was used to compare the differences between two groups. Categorical variables were presented as number and percentage, and compared using Chi-square test or Fisher's exact test when appropriate. A p-value less than 0.05 was considered as statistically significant difference, and all tests were two-sided. Data were analyzed using SPSS version 23.0 (IBM Corp., Armonk, NY, USA).

\section{Results}

\section{Demographic and surgical characteristics}

From 2,748 patients who undertook TKA in our hospital between January 2014 to December 2019, 560 patients with unilateral TKA and 285 patients with bilateral TKA were selected following the inclusion and exclusion criteria. Among unilateral TKA patients, 180 used aspirin preoperatively while 380 patients did not. Among bilateral TKA patients, 100 used aspirin and 185 patients did not (Fig. 1). Of the 280 patients used aspirin preoperatively, $226(80.71 \%)$ took 100 mg daily and 54 (19.29\%) took 80 mg daily. And 157 (56.07\%) patients used aspirin for primary prevention while $123(43.93 \%)$ for secondary prevention.

For demographic characteristics and medical conditions, in both unilateral and bilateral TKA series, there were no significant differences between the aspirin and non-aspirin group regarding age, sex, $\mathrm{BMI}$, knee deformity and tobacco/alcohol use $(p>0.05)$. In terms of comorbidities, aspirin group had higher proportion of CAD $(p<0.01)$ than the control group. No significant differences were detected in the proportions of hypertension, diabetes, stroke or arrhythmia between aspirin and non-aspirin group in both unilateral and bilateral TKAs $(p>0.05)$ (Table 1). For surgical characteristics, there was no significant difference in anesthesia, implant types or tourniquet time, either $(p>0.05)$ (Table 2). 
Table 1

Demographic and medical conditions of the included patients

\begin{tabular}{|c|c|c|c|c|c|c|}
\hline & \multicolumn{3}{|c|}{ Unilateral TKA $(\mathrm{N}=560)$} & \multicolumn{3}{|c|}{ Bilateral TKA $(\mathrm{N}=285)$} \\
\hline & Aspirin & Non-aspirin & $P$ value & Aspirin & Non-aspirin & $P$ value \\
\hline Number & 180 & 380 & - & 100 & 185 & - \\
\hline Age (yr) & $70.39(5.94)$ & $69.62(6.54)$ & 0.181 & $68.84(7.93)$ & $68.75(6.74)$ & 0.793 \\
\hline \multicolumn{7}{|l|}{ Sex } \\
\hline Female & $136(75.56)$ & $285(75.00)$ & 0.887 & $78(78.00)$ & $139(75.14)$ & 0.446 \\
\hline Male & $44(24.44)$ & $95(25.00)$ & & $22(22.00)$ & $46(24.86)$ & \\
\hline $\mathrm{BMI}(\mathrm{Kg} / \mathrm{m} 2)$ & $27.18(3.21)$ & $27.82(4.03)$ & 0.262 & $27.40(2.17)$ & $27.31(3.40)$ & 0.811 \\
\hline Knee deformity & $47(26.11)$ & $94(24.74)$ & 0.726 & $26(26.00)$ & $47(25.41)$ & 0.913 \\
\hline \multicolumn{7}{|l|}{ Comorbidity } \\
\hline Hypertension & $143(79.44)$ & $279(73.42)$ & 0.122 & $66(66.00)$ & $133(71.89)$ & 0.301 \\
\hline Diabetes & $58(32.22)$ & $148(38.94)$ & 0.123 & $30(30.00)$ & $73(39.46)$ & 0.113 \\
\hline CAD & $86(46.24)$ & $126(33.16)$ & 0.005 & $51(51.00)$ & $50(27.03)$ & $<0.001$ \\
\hline Stroke* & $42(23.33)$ & $73(19.21)$ & 0.259 & $11(11.00)$ & $29(15.68)$ & 0.278 \\
\hline Arrhythmia & $25(13.89)$ & $36(9.47)$ & 0.117 & $8(8.00)$ & $7(3.78)$ & 0.143 \\
\hline Tobacco use & $15(8.33)$ & $33(8.69)$ & 0.890 & $7(7.00)$ & $21(9.72)$ & 0.212 \\
\hline Alcohol use & $9(5.00)$ & $26(6.84)$ & 0.400 & $5(5.00)$ & $7(3.78)$ & 0.626 \\
\hline \multicolumn{7}{|c|}{ * Include transient ischemic attack. } \\
\hline \multicolumn{7}{|c|}{ BMI: Body mass index; CAD: Coronary artery disease; } \\
\hline \multicolumn{7}{|c|}{ Values are given as the mean, with the standard deviation in parentheses (Age, BMI). } \\
\hline
\end{tabular}


Table 2

Surgical characteristics of the included patients

\section{Unilateral TKA $(\mathrm{N}=560) \quad$ Bilateral TKA $(\mathrm{N}=285)$}

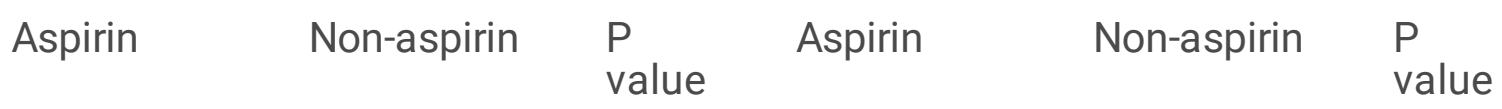

\begin{tabular}{lllllll} 
Anesthesia & & & & & \\
General & $170(94.44)$ & $344(90.53)$ & 0.115 & $99(99.00)$ & $180(97.30)$ & 0.601 \\
Spinal & $10(5.56)$ & $36(9.47)$ & & $1(1.00)$ & $5(2.70)$ & \\
Implant style & & & & & & \\
PS & $135(75.00)$ & $298(78.42)$ & 0.367 & $94(94.00)$ & $173(93.51)$ & 0.872 \\
CR & $45(25.00)$ & $82(21.58)$ & & $6(6.00)$ & $12(6.49)$ & \\
$\begin{array}{l}\text { Tourniquet } \\
\text { time* }\end{array}$ & $\begin{array}{l}81.02 \\
(12.93)\end{array}$ & $\begin{array}{l}81.97 \\
(14.50)\end{array}$ & 0.454 & $\begin{array}{l}82.57 \\
(13.18)\end{array}$ & $\begin{array}{l}80.70 \\
(17.98)\end{array}$ & 0.731 \\
\hline
\end{tabular}

PS: Posterior stabilization; CR: Cruciate retention; LCCK: Legacy constrained condylar knee.

Values are given as the mean, with the standard deviation in parentheses (Tourniquet time).

Values are given as the number of patients, with the percentage in parentheses (Anesthesia, implant type).

*Tourniquet time for one side.

\section{Primary Outcomes}

For blood loss during the surgery, they were similar between the aspirin and non-aspirin group in both unilateral and bilateral TKA $(63.88 \mathrm{ml}$ vs. $63.94 \mathrm{ml}$ for unilateral TKAs, and $115.45 \mathrm{ml}$ vs. $110.79 \mathrm{ml}$ for bilateral TKAs, $p>0.05$ respectively). The amount of drainage was also monitored after TKA. The drainage volumes were both higher in the aspirin group than the non-aspirin group in unilateral and bilateral TKAs (229.17 ml vs. $211.28 \mathrm{ml}$ for unilateral TKAs, and $456.21 \mathrm{ml}$ vs. $402.54 \mathrm{ml}$ for bilateral TKAs). However, only in bilateral TKAs was the difference statistically significant $(p<0.05)$. For transfusion, neither of the groups had transfusions during unilateral TKAs and there was no significant difference in transfusion rate during bilateral TKAs $(3.00 \%$ vs. $2.70 \%, p>0.05)$. The transfusion rates after the surgeries did not show any significant differences, either (4.44\% vs. $4.74 \%$ for unilateral TKAs, and $11.00 \%$ vs. $10.27 \%$ for bilateral TKAs, $p>0.05$ respectively) (Table 3 ). 
Table 3

Comparisons of blood loss, drainage and transfusion rate between the aspirin and non-aspirin group

\begin{tabular}{|c|c|c|c|c|c|c|}
\hline & \multicolumn{2}{|c|}{ Unilateral TKA $(\mathrm{N}=560)$} & \multicolumn{4}{|c|}{ Bilateral TKA $(\mathrm{N}=285)$} \\
\hline & Aspirin & Non-aspirin & $\begin{array}{l}\mathrm{P} \\
\text { value }\end{array}$ & Aspirin & $\begin{array}{l}\text { Non- } \\
\text { aspirin }\end{array}$ & $\begin{array}{l}\mathrm{P} \\
\text { value }\end{array}$ \\
\hline \multirow{2}{*}{$\begin{array}{l}\text { Blood loss during the } \\
\text { surgery }\end{array}$} & 63.88 & 63.94 & \multirow[t]{2}{*}{0.986} & \multirow{2}{*}{$\begin{array}{l}115.45 \\
(60.21)\end{array}$} & 110.79 & \multirow[t]{2}{*}{0.427} \\
\hline & $(30.85)$ & $(39.13)$ & & & $(38.48)$ & \\
\hline Transfusion 1 & $0(0.00)$ & $0(0.00)$ & - & $3(3.00)$ & $5(2.70)$ & 1.000 \\
\hline \multirow[t]{2}{*}{ Drainage } & 229.17 & \multirow{2}{*}{$\begin{array}{l}211.28 \\
(135.36)\end{array}$} & \multirow[t]{2}{*}{0.184} & 456.21 & 402.54 & \multirow[t]{2}{*}{0.043} \\
\hline & $(150.24)$ & & & $(230.14)$ & $(201.88)$ & \\
\hline Transfusion 2 & $8(4.44)$ & $18(4.74)$ & 0.878 & $11(11.00)$ & $\begin{array}{l}19 \\
(10.27)\end{array}$ & 0.848 \\
\hline \multicolumn{7}{|c|}{ Blood loss: Blood loss during the surgery. } \\
\hline \multicolumn{7}{|c|}{ Transfusion 1: Transfusion during the surgery. } \\
\hline \multicolumn{7}{|c|}{ Transfusion 2: Transfusion after the surgery. } \\
\hline \multicolumn{7}{|c|}{ Values are given as the mean, with the standard deviation in parentheses (Tourniquet time). } \\
\hline $\begin{array}{l}\text { Values are given as th } \\
\text { type). }\end{array}$ & per of pat & s, with the & tage & arenthes & sthes & lant \\
\hline
\end{tabular}

HGB and HCT level could indirectly reflect the blood loss in patients, therefore HGB and HCT level before and after the surgeries were also compared. First, the levels of HGB and HCT before the surgeries were not different in the two groups for both unilateral and bilateral TKA series $(p>0.05)$. Then, the HGB and HCT levels at POD 1, 2, 3, 5 until patient discharge were compared. The results showed that there were no differences between aspirin and non-aspirin groups at POD 1, 2, 3 in both unilateral and bilateral TKA ( $p>$ 0.05), while at POD5, the HGB and HCT level in aspirin group were significant lower not only in unilateral TKAs (104.15g/L vs. $107.49 \mathrm{~g} / \mathrm{L}$ for HGB, and $30.89 \%$ vs. $32.70 \%$ for HCT, $p<0.05$ respectively), but also in bilateral TKAs (95.39g/L vs. $99.18 \mathrm{~g} / \mathrm{L}$ for HGB, and $27.79 \%$ vs. $29.91 \%$ for HCT, $p<0.05$ respectively). (Fig. 2).

\section{Secondary Outcomes}

Besides blood loss, complications were also important outcomes. For total complication rates, there were no significant differences between the aspirin groups and non-aspirin groups either for both unilateral and bilateral TKA series ( $6.11 \%$ vs. $9.74 \%$ for unilateral TKAs, and $8.00 \%$ vs. $9.73 \%$ for bilateral TKAs, $p>$ 0.05 respectively). For vascular related complications (including cardiac events, VTE events and cerebrovascular events), the rate was $3.33 \%$ for aspirin group, which was significant lower than that of 
non-aspirin group $(7.89 \%, p<0.05)$. No significant difference was found in bilateral TKA series $(6.00 \%$ vs. $8.65 \%, p>0.05)$. For wound related complication rates, there were not significant different between the two groups $(2.22 \%$ vs. $0.79 \%$ for unilateral TKA, and $2.00 \%$ vs. $0.54 \%$ for bilateral TKA, $p>0.05$ respectively).

We further compare the proportions of various complications, which were classified into cardiac events, cerebrovascular events, VTE events, wound events and others. For unilateral TKAs, the proportion of wound events of aspirin group was significant higher than that of non-aspirin group ( $36.36 \% \mathrm{vs.} 7.50 \%, p$ $<0.05)$, while other rates were similar $(p>0.05)$. For bilateral TKAs, no significant differences were found between the two groups $(p>0.05)$. Although the complication rates were different, the length of stay between the two groups in unilateral and bilateral TKA were similar (10.93d vs. $10.98 \mathrm{~d}$ for unilateral TKA, and $11.55 \mathrm{~d}$ vs. $11.99 \mathrm{~d}$ for bilateral TKA, $p>0.05$ respectively) (Table 4 ). 
Table 4

Comparisons of complication rate between the aspirin and non-aspirin group

\begin{tabular}{|c|c|c|c|c|c|c|}
\hline & \multicolumn{3}{|c|}{ Unilateral TKA $(N=560)$} & \multicolumn{3}{|c|}{ Bilateral TKA $(\mathrm{N}=285)$} \\
\hline & Aspirin & $\begin{array}{l}\text { Non- } \\
\text { aspirin }\end{array}$ & $\begin{array}{l}\mathrm{P} \\
\text { value }\end{array}$ & Aspirin & Non-aspirin & $\begin{array}{l}P \\
\text { value }\end{array}$ \\
\hline Complications* & $11(6.11)$ & 37 (9.74) & 0.152 & $8(8.00)$ & $18(9.73)$ & 0.628 \\
\hline Vascular related & $6(3.33)$ & $30(7.89)$ & 0.040 & $6(6.00)$ & $16(8.65)$ & 0.424 \\
\hline Wound related & $4(2.22)$ & $3(0.79)$ & 0.309 & $2(2.00)$ & $1(0.54)$ & 0.586 \\
\hline Total events ${ }^{\star \star}$ & 11 & 40 & - & 9 & 19 & - \\
\hline Cardiac events & $2(18.18)$ & $10(25.00)$ & 0.944 & $2(22.22)$ & $5(26.32)$ & 1.000 \\
\hline $\begin{array}{l}\text { Cerebrovascular } \\
\text { events }\end{array}$ & $1(9.09)$ & $5(12.50)$ & 1.000 & $1(11.11)$ & $3(15.79)$ & 1.000 \\
\hline VTE events & $3(27.27)$ & $17(42.50)$ & 0.570 & $4(44.44)$ & $8(42.11)$ & 1.000 \\
\hline Wound events & $4(36.36)$ & $3(7.50)$ & 0.049 & $2(22.22)$ & $1(5.26)$ & 0.234 \\
\hline Others ${ }^{\star \star *}$ & $1(9.09)$ & $5(12.50)$ & 1.000 & $0(0.00)$ & $2(10.53)$ & - \\
\hline Length of stay & $\begin{array}{l}10.93 \\
(4.93)\end{array}$ & $\begin{array}{l}10.98 \\
(5.50)\end{array}$ & 0.917 & $\begin{array}{l}11.55 \\
(4.64)\end{array}$ & $\begin{array}{l}11.99 \\
(4.66)\end{array}$ & 0.447 \\
\hline \multicolumn{7}{|c|}{$\begin{array}{l}\text { VTE: venous thromboemlolism, including venous thromboemlolism and pulmonary } \\
\text { thromboembolism. }\end{array}$} \\
\hline \multicolumn{7}{|c|}{ *Complications were counted by patients. } \\
\hline \multicolumn{7}{|c|}{ ** Different evens were counted by cases. } \\
\hline \multicolumn{7}{|c|}{$\star \star \star$ Include infection and implant related complication. } \\
\hline \multicolumn{7}{|c|}{ Values are given as the mean, with the standard deviation in parentheses (Length of stay). } \\
\hline
\end{tabular}

\section{Discussion}

Aspirin is one of the commonly prescribed medications worldwide [5]. There are a growing number of patients who take aspirin routinely before TKA. However, it remains controversial regarding the safety of continuing use of aspirin during the perioperative period of TKA. Schwab et al conducted a retrospective study of unilateral TKA to compare 154 knees with aspirin and 301 knees without aspirin in 2015. They found that there were no differences in terms of blood loss as measured by HGB drop and transfusion rate between the two groups [9]. Other studies also looked into this topic in recent years [10-12]. However, 
there were some limitations in these studies. Firstly, the patients were not matched well at baseline, including age, BMI, and underlying diseases, which may introduce bias in the comparisons $[9,12]$. Secondly, some studies did not compare the complication rate of the two groups which is an important outcome $[9,10]$. Thirdly, most of the studies did not perform the comparisons in bilateral TKA which may have higher risk in terms of blood loss and complication rate because of the longer surgical time and greater trauma $[9-11,16]$. Therefore, the present study aimed to compare blood loss, transfusion rate and complication rate of the unilateral and bilateral TKA with or without aspirin under more consistent criteria.

In order to reduce bias, stringent inclusion and exclusion criteria were applied to choose the aspirin and non-aspirin groups. For TKA, the indications included almost all end-stage knee joint diseases, such as osteoarthritis, rheumatoid arthritis (RA), and hemophilic arthritis (HA). Among these, osteoarthritis is no doubt the leading cause which is a chronic degenerative joint disease closely related with aging $[2,17]$. Thus, for the consistency of age and joint diseases, only osteoarthritis patients in our database were included. Then patients with the risk of vascular events according to the ACC/AHA guideline were selected from osteoarthritis patients to guarantee the consistency between aspirin group and control group [13]. In order to decrease the bias in blood loss estimation, all included cases used tourniquet and drainage. As a result, the demographic and surgical characteristics between the two groups were largely comparable. There were no differences in age, sex, BMI, knee deformity, tobacco/alcohol use, anesthesia or implant type. However, there were also certain differences in morbidities, namely the proportions of CAD due to the selection bias by aspirin use which was hard to eliminate $[7,18]$.

In standard procedure of TKA, it needs at least 6 times of osteotomy and has a large cancellous bone cutting surface which would cause relatively high blood loss. It was reported that a total calculated blood loss could reach $1,000 \mathrm{ml}$ in unilateral TKA, and it would be more in bilateral TKA $[16,19]$. For the bleeding risk, we detected various indicators, including blood loss and transfusion rate during the surgery, drainage and transfusion rate after the surgery, HGB and HCT change during the perioperative period. Although the transfusion rates were similar, the drainage volumes were higher in the aspirin group than the non-aspirin group, especially in bilateral TKAs (17 ml higher in unilateral TKA and $54 \mathrm{ml}$ higher in bilateral TKA). It also reflects in the HGB and HCT levels after surgery. Both the HGB and HCT level in aspirin group were significant lower at POD5.

Aspirin may also influence the complication rate during the perioperative period of TKA. On one hand, aspirin could reduce the risk of various vascular events $[7,18]$. In the present study, after matching the vascular risk of the two groups under the ACC/AHA guideline, we found the rate of vascular related complications (including cardiac events, VTE events and cerebrovascular events) in aspirin group was significant lower than that of non-aspirin group in unilateral TKAs. On the other hand, aspirin may increase the risk of wound complications of TKA. The soft tissue around the TKA incision is relatively thin so that it is vulnerable to various factors, such as bleeding in the incision edges [20]. For example, the wound complication rate was much higher in the HA patients who had coagulation disorder [22]. Whether the use of aspirin could influence wound healing in TKA remains unknown. In the present study, we compare the rate of wound complication in the two groups. Although there were not significant 
differences in the complication rate, but wound events occupied a significant higher proportion in complications of aspirin group than that of non-aspirin group (36.36\% vs. $7.50 \%)$. Among the 4 wound events of aspirin group, one was surgical site infection and others were poor wound healing, which indicated the aspirin use might affect the wound healing. It draws the attention regarding adverse effects of aspirin usage on wound healing, especially for patients with high risk of wound events, such as patients with RA or post-traumatic arthritis $[14,23]$.

The present study thoroughly detected the bleeding risk and complication rate under continuing use of aspirin in both unilateral and bilateral TKA. It is also a relatively large study which included 280 patients who used aspirin during the perioperative period [9-12]. The main limitation of the present study was the retrospective design, and our findings need to be validated by future perspective studies with larger sample sizes.

\section{Conclusion}

Continuing use of aspirin could prevent vascular related complications during the perioperative period of TKA. However, it might also increase the risk of bleeding and wound complications which we should pay attention to.

\section{Abbreviations}

TKA: total knee arthroplasty; ACC: American College of Cardiology; AHA: American Heart Association; CAD: coronary artery diseases; HGB: hemoglobin; HCT: hematocrit; SD: standard deviation; BMI: body mass index; RA: rheumatoid arthritis; HA: hemophilic arthritis.

\section{Declarations}

\section{Acknowledgements}

We would like to give our sincere appreciation to Prof. Jin Lin, Prof. Jin Jin, Prof. Wenwei Qian, Dr. Yingjie Wang, Dr. Chang Han and Dr. Yongbo Xiang for their helpful contributions on this article.

\section{Authors' contributions}

$X W$ and ZL designed the study. ZL, SX and YD performed data acquisition. YD and BF performed statistical analyses. ZL drafted the manuscript. XW revised the manuscript critically. All authors reviewed and approved the final manuscript.

\section{Funding}

This study was supported by grants from the National Natural Science Foundation of China (81871786, 81871740) and Medical Science and Technology Research Fund of Guangdong Province (B2021165). 
Availability of data and materials

The original data and materials are available from the corresponding author on reasonable request.

\section{Ethics approval and consent to participate}

This study was approved by the Institutional Review Board of Peking Union Medical College Hospital. The need for informed consent was waived because this was a retrospective analysis of data from the hospital database.

\section{Consent for publication}

Not applicable.

\section{Competing interests}

The authors declare that they have no competing interests.

\section{References}

1. Pisanu F, Andreozzi M, Costagli F, Caggiari G, Saderi L, Sotgiu G, et al. Resumption of physical activity and sport after knee replacement. J Arthroplasty 2020;20:247-50.

2. Choi HG, Kwon BC, Kim JI, Lee JK. Total knee arthroplasty reduces the risk of mortality in osteoarthritis patients up to 12 years: A Korean national cohort longitudinal follow-up study. J Orthop Surg (Hong Kong) 2020;28:2309499020902589.

3. Passias PG, Bono OJ, Bono JV. Total Knee Arthroplasty in Patients of Advanced Age: A Look at Outcomes and Complications. J Knee Surg 2018;33:1-7.

4. Steg PG, Bhatt DL, Simon T, Fox K, Mehta SR, Harrington RA, et al. Ticagrelor in Patients with Stable Coronary Disease and Diabetes.N Engl J Med 2019;381:1309-1320.

5. Stuntz M, Bernstein B. Recent trends in the prevalence of low- dose aspirin use for primary and secondary prevention of cardiovascular disease in the United States, 2012-2015. Prev Med Rep 2017;5:183-6.

6. Donadini MP, Bellesini M. Aspirin Plus Clopidogrel vs Aspirin Alone for Preventing Cardiovascular Events Among Patients at High Risk for Cardiovascular Events. JAMA 2018;320:593-4.

7. Schamroth Pravda M, Schamroth Pravda N, Beigel Y, Matetzky S, Beigel R. The Aspirin Primary Prevention Conundrum. Isr Med Assoc J 2020;22:60-3

8. Armstrong MJ, Gronseth G, Anderson DC, Biller J, Cucchiara B, Dafer R, et al. Summary of evidencebased guideline: periprocedural management of antithrombotic medications in patients with ischemic cerebrovascular disease: report of the Guideline Development Subcommittee of the American Academy of Neurology. Neurology 2013;80:2065-9 
9. Schwab PE, Lavand'homme P, Yombi J, Thienpont E. Aspirin mono-therapy continuation does not result in more bleeding after knee arthroplasty. Knee Surg Sports Traumatol Arthrosc 2017;25:258693.

10. Hang G, Chen JY, Yew AKS, Pang HN, Jin DTK, Chia SL, Lo NN, Yeo SJ (2020) Effects of continuing use of aspirin on blood loss in patients who underwent unilateral total knee arthroplasty. $\mathrm{J}$ Orthop Surg (Hong Kong) 28(1):2309499019894390.

11. Meier R, Marthy R, Saely CH, Kuster MS, Giesinger K, Rickli H. Comparison of preoperative continuation and discontinuation of aspirin in patients undergoing total hip or knee arthroplasty. Eur J Orthop Surg Traumatol 2016;26:921-8.

12. Chen CF, Tsai SW, Wu PK, Chen CM, Chen WM. Does continued aspirin mono-therapy lead to a higher bleeding risk after total knee arthroplasty? J Chin Med Assoc 2019;82:60-5.

13. Eagle KA, Berger PB, Calkins H, Chaitman BR, Ewy GA, Fleischmann KE, et al. ACC/AHA guideline update for perioperative cardiovascular evaluation for noncardiac surgery- executive summary a report of the American College of Cardiology/American Heart Association Task Force on Practice Guidelines. Circulation 2002;105:1257-67.

14. Vakharia AM, Cohen-Levy WB, Vakharia RM, Sodhi N, Mont MA, Roche MW. Perioperative Complications in Patients with Rheumatoid Arthritis Following Primary Total Knee Arthroplasty: An Analysis of 102,898 Patients. J Knee Surg 2019;32:1075-80.

15. Singh JA. Total knee arthroplasty outcomes in lupus: a study using the US National Inpatient Sample. Rheumatology (Oxford) 2019;58:2130-36.

16. Richardson SS, Kahlenberg CA, Blevins JL, Goodman SM, Sculco TP, Figgie MP, et al. Complications associated with staged versus simultaneous bilateral total knee arthroplasty: An analysis of 7747 patients. Knee 2019;26:1096-101.

17. Hunter DJ. Osteoarthritis. Lancet 2019;393:1745-59.

18. Verheugt FWA, Ten Berg JM, Storey RF, Cuisset T, Granger CB. Antithrombotics: From Aspirin to DOACs in Coronary Artery Disease and Atrial Fibrillation. J Am Coll Cardiol 2019;74:699-711.

19. Kapadia BH, Torre BB, Ullman N, Yang A, Harb MA, Grieco PW, et al. Reducing perioperative blood loss with antifibrinolytics and antifibrinolytic-like agents for patients undergoing total hip and total knee arthroplasty. J Orthop 2019;16:513-6.

20. Gupta VK. Pretubercular Subcutaneous Thickness Is a Protective Factor for Superficial Wound Complications After Total Knee Arthroplasty in Nonmorbidly Obese Patients. J Arthroplasty 2020;35:255-8.

21. Song SJ, Bae JK, Park CH, Yoo MC, Bae DK, Kim KI. Mid-term outcomes and complications of total knee arthroplasty in haemophilic arthropathy: A review of consecutive 131 knees between 2006 and 2015 in a single institute. Haemophilia 2018;24:299-306.

22. Rodriguez-Merchan EC. The knee in severe haemophilia with special emphasis on surgical/invasive procedures. Thromb Res 2014;134:545-51. 
23. Brockman BS, Maupin JJ, Thompson SF, Hollabaugh KM, Thakral R. Complication Rates in Total Knee Arthroplasty Performed for Osteoarthritis and Post-Traumatic Arthritis: A Comparison Study. J Arthroplasty 2020;35:371-4.

\section{Figures}

Primary total knee arthroplasty from the database $(\mathrm{N}=2,748)$

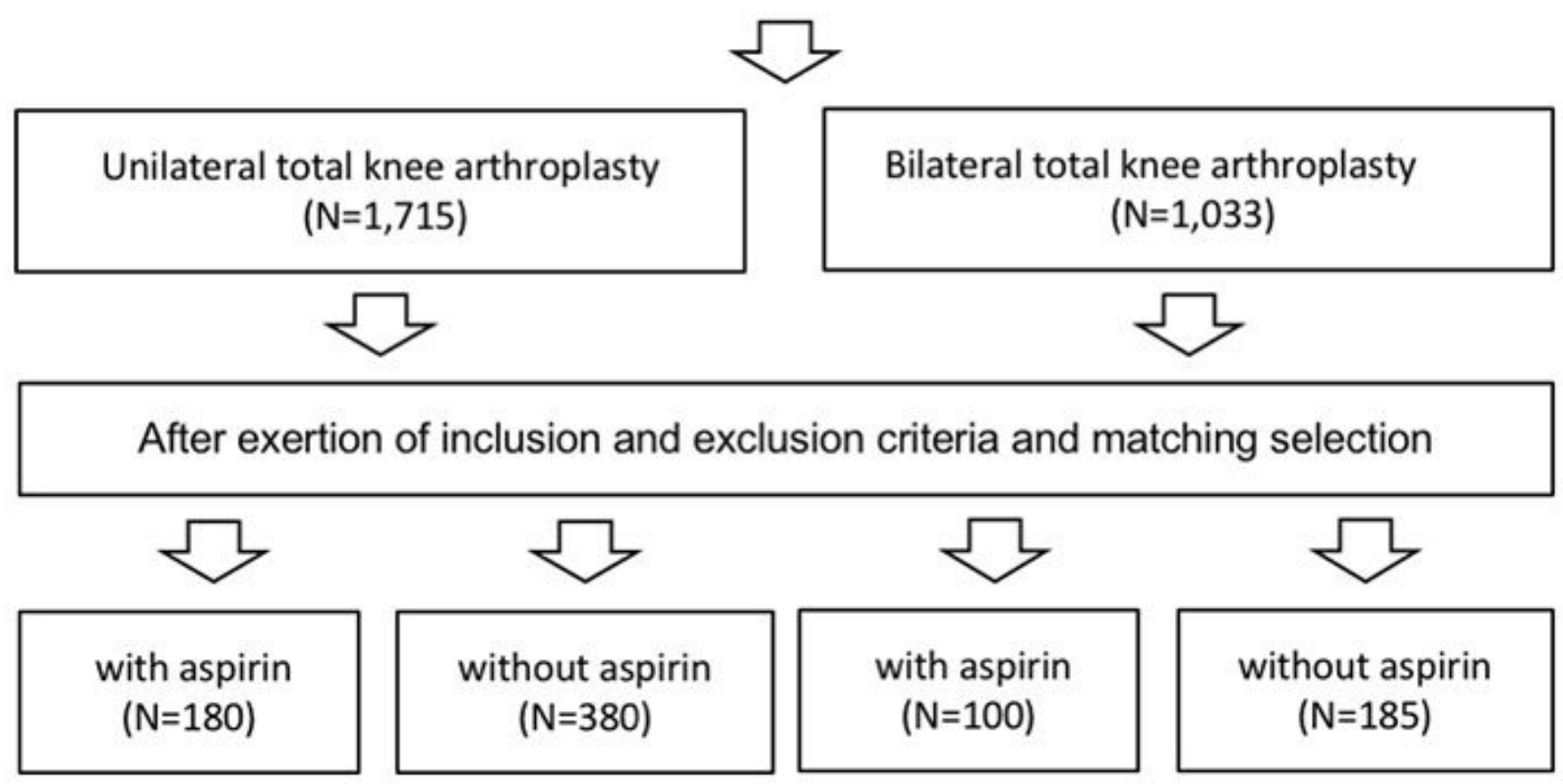

Figure 1

Flowchart of the study participants. 


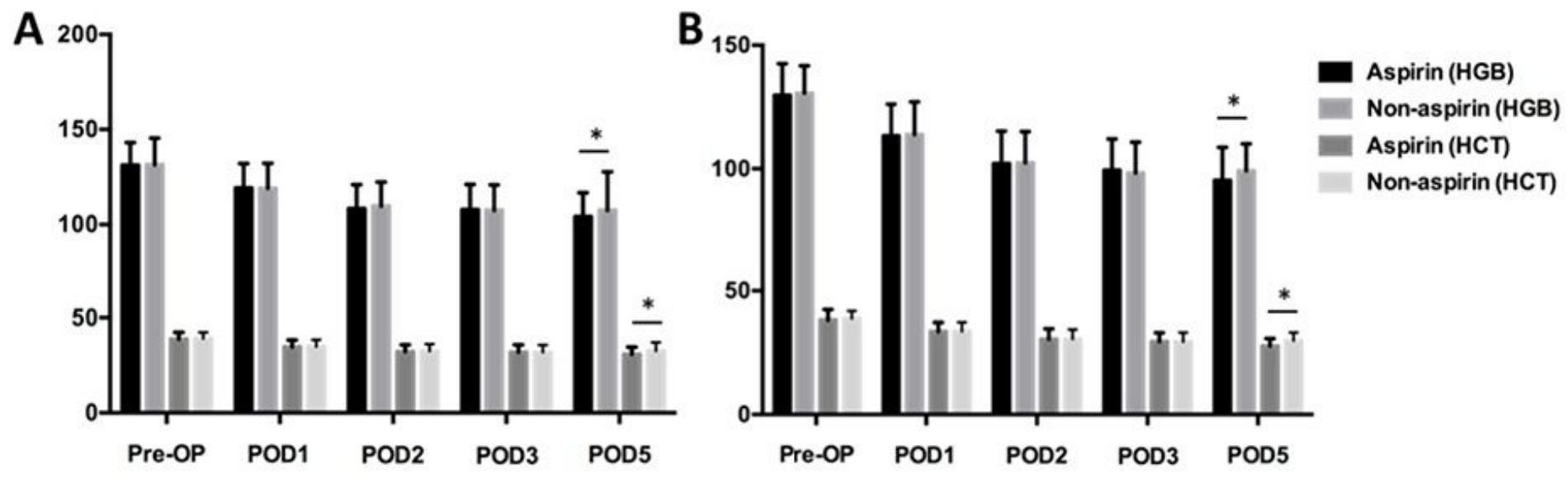

Figure 2

Perioperative HGB and HCT levels of TKA patients in aspirin group and non-aspirin group. A.

Perioperative HGB and HCT levels of unilateral TKA patients. B. Perioperative HGB and HCT levels of

bilateral TKA patients. HGB: hemoglobin; HCT: hematocrit; POD: postoperative day. ( $\left.{ }^{*} p<0.05\right)$ 